\title{
\begin{tabular}{l|l} 
Mitraries & DSpace@MIT
\end{tabular}
}

\author{
MIT Open Access Articles
}

\section{Feasibility Study for Elimination of the Screening Current-Induced Fields in HTS Coil}

The MIT Faculty has made this article openly available. Please share how this access benefits you. Your story matters.

Citation: Kim, K. L. et al. “Feasibility Study for Elimination of the Screening Current-Induced Fields in HTS Coil." Journal of Superconductivity and Novel Magnetism 28.1 (2015): 83-88.

As Published: http://dx.doi.org/10.1007/s10948-014-2790-y

Publisher: Springer US

Persistent URL: http://hdl.handle.net/1721.1/105214

Version: Author's final manuscript: final author's manuscript post peer review, without publisher's formatting or copy editing

Terms of Use: Article is made available in accordance with the publisher's policy and may be subject to US copyright law. Please refer to the publisher's site for terms of use. 


\title{
Feasibility Study for Elimination of the Screening Current-Induced Fields in HTS Coil
}

\author{
K. L. Kim • S. Hahn • Y. H. Choi • Y. G. Kim • \\ D. H. Kang • K. Kazikawa $\cdot$ H. G. Lee
}

Received: 29 July 2014 / Accepted: 7 September 2014 / Published online: 29 November 2014

(C) Springer Science+Business Media New York 2014

\begin{abstract}
Herein, we report a feasibility study for elimination of the screening current-induced field (SCIF) in fully insulated (INS) and no-insulation (NI) GdBCO coated conductor (CC) coils exposed to an external AC magnetic field generated by background solenoid copper coils. Prior to investigating the effects of external $\mathrm{AC}$ magnetic fields on SCIF in the GdBCO CC coils, the magnetic flux density $\left(B_{z}\right)$ was calculated using the equivalent circuit model and compared to the $B_{z}$ obtained empirically to quantify the SCIF in the INS and NI coils. The value of the SCIF in the NI coil was smaller than that in the INS coil without the use of an external AC magnetic field, due to the current paths originating from the turn-to-turn and layer-to-layer contacts, suggesting that the screening current dissipated by contact resistance during charging. When the INS and NI coils were exposed to the external AC magnetic field, the $B_{z}$ of the coils increased gradually, and eventually saturating to the calculated $B_{z}$ values, indicating full removal of the SCIF. In addition, the SCIF of the NI coil could be removed under subjection to a lower external AC magnetic field compared to the INS coil, due to the lower SCIF occurring in the NI coil after charging.
\end{abstract}

K. L. Kim · Y. H. Choi · Y. G. Kim · D. H. Kang · H. G. Lee ( $ه)$

Department of Materials Science and Engineering,

Korea University, Seoul, South Korea

e-mail: haigunlee@korea.ac.kr

S. Hahn

Francis Bitter Magnet Laboratory, Massachusetts Institute of Technology, Cambridge, MA 02139, USA

K. Kazikawa

Institute of Superconductor Science and Systems, Kyushu

University, Fukuoka, 812-8581, Japan
Keywords GdBCO CC $\cdot$ No-insulation $\cdot$ Screening current-induced field $\cdot$ Solenoid coil $\cdot$ Vortex shaking effect

\section{Introduction}

The second generation (2G) high-temperature superconductor (HTS) coated conductor (CC) shows promise for superconducting applications because of its high critical current $\left(I_{c}\right)$ density, and the low dependency of $I_{c}$ on the external magnetic field compared to low temperature counterparts. Therefore, magnets fabricated with 2G HTS CC can only be used for the development of ultra-high-field magnets that will be used for nuclear magnetic resonance (NMR) conducted beyond $1 \mathrm{GHz}$ [1-4]. However, during charging of the HTS magnet, the magnetic flux can penetrate into the vortex at above a certain critical field strength, which causes a screening current to circulate within the HTS even after complete discharge of the magnet. The screening current generates a remnant magnetic field, termed the screening current-induced field (SCIF), and exists until the HTS magnet loses its superconductivity [5-8]. Such an undesirable remnant field leads to detrimental effects on NMR performance, such as spatial homogeneity and temporal stability of the magnetic field within a target volume at the magnet's center, which is a major hindrance to the practical utilization of HTS in high-resolution NMR.

Recently, the no-insulation (NI) winding technique has been actively studied for the development of magnets with greatly enhanced mechanical integrity and thermal stability [9-16]. This technique can be applied to HTS to achieve reduced weight, size, and consumption of the conductor, especially for use in NMR spectroscopy as an NMR magnet generating an extremely high field. According to recent studies, the SCIF of the 2 G HTS magnet, which is 
conventionally insulated every turn, can be effectively removed by exposing the magnet to an external AC magnetic field $[17,18]$. When the flat surface of the HTS CC lay in parallel to an external AC magnetic field, the direction of the magnetization changed from the radial direction to the axial direction, the so-called vortex shaking effect [19-22]. Consequently, the changed direction of magnetization in the HTS CC cannot affect the center field of the magnet being charged at the target current.

In this study, the effect of an external AC magnetic field on the elimination of SCIF in both fully insulated (INS) and NI coils was clearly investigated by measuring the magnetic field at the center of the coils $\left(B_{z}\right)$ with respect to the amplitude of the AC currents in background coils wound with copper wires.

\section{Experimental Setup}

Table 1 lists the design parameters of the solenoid test coils fabricated using $\mathrm{Cu}$-stabilized GdBCO coated conductor (SuNAM Co., Ltd.), to a width of 4.1-mm and a thickness of 0.1-mm. In this study, two types of GdBCO CC coils were fabricated: one wound with Kapton tape (INS), and the other wound without turn-to-turn insulation (NI). Both coils had similar configurations, indicating similar coil constants and self-inductances. The critical current $\left(I_{c}\right)$ values of the INS and NI coils were measured at $77 \mathrm{~K}$ using a criterion of $1 \mu \mathrm{V} / \mathrm{cm}$ to be 167 and $170 \mathrm{~A}$, respectively. The background

Table 1 Design parameters of solenoid test coils

\begin{tabular}{|c|c|c|c|}
\hline Parameters & & INS coil & NI coil \\
\hline \multicolumn{4}{|l|}{ Conductor } \\
\hline Width; thickness & {$[\mathrm{mm}]$} & \multicolumn{2}{|c|}{$4.1 ; 0.1$} \\
\hline Stabilizer thickness & {$[\mu \mathrm{m}]$} & \multicolumn{2}{|c|}{$32 \sim 50$} \\
\hline Ag layer thickness & {$[\mu \mathrm{m}]$} & \multicolumn{2}{|c|}{$0.9 \sim 1.1$} \\
\hline GdBCO layer thickness & {$[\mu \mathrm{m}]$} & \multicolumn{2}{|c|}{$0.9 \sim 1.1$} \\
\hline Buffer layer thickness & {$[\mu \mathrm{m}]$} & \multicolumn{2}{|c|}{$0.085 \sim 0.105$} \\
\hline Substrate thickness & {$[\mu \mathrm{m}]$} & \multicolumn{2}{|c|}{$58 \sim 62$} \\
\hline \multicolumn{4}{|l|}{ Coil } \\
\hline Insulation method & & Turn-to-turn & No insulation \\
\hline Insulation material & & Kapton & N/A \\
\hline Total length of the CC used & {$[\mathrm{m}]$} & 7.95 & 7.89 \\
\hline Number of turns & & 15 & 15 \\
\hline Number of layers & & 3 & 3 \\
\hline Inner; outer diameter & {$[\mathrm{mm}]$} & $65 ; 67$ & $65 ; 65.5$ \\
\hline Coil height & {$[\mathrm{mm}]$} & 60 & 60 \\
\hline Coil constant ( $\left.B_{\mathrm{z}} @ 1 \mathrm{~A}\right)$ & [mT/A] & 0.6340 & 0.6379 \\
\hline Self-inductance & {$[\mu \mathrm{H}]$} & 95.4 & 94.6 \\
\hline$I_{\mathrm{c}} @ 77 \mathrm{~K}$, self-field & {$[\mathrm{A}]$} & 167 & 710 \\
\hline
\end{tabular}

Table 2 Specifications of the BG coils

\begin{tabular}{lll}
\hline Parameters & & Inner/outer BG coils \\
\hline Winding direction & & Clockwise/counterclockwise \\
Diameter of copper wire & {$[\mathrm{mm}]$} & 1 \\
Total length of the wire used & {$[\mathrm{m}]$} & $5.65 / 9.61$ \\
Number of turns & & 180 \\
Number of layers & & 2 \\
Inner; outer diameter & {$[\mathrm{mm}]$} & $50 ; 54 / 85 ; 89$ \\
Coil height & {$[\mathrm{mm}]$} & 180 \\
Coil constant $\left(B_{\mathrm{Z}}\right.$ at $\left.1 \mathrm{~A}\right)$ & {$[\mathrm{mT} / \mathrm{A}]$} & $2.4145 / 2.2628$ \\
Self-inductance & {$[\mu \mathrm{H}]$} & $166 / 435$ \\
\hline
\end{tabular}

copper coils (inner/outer BG coils) consisted of 180 turns and 2 layers of copper wires with a diameter of $1 \mathrm{~mm}$. The specifications of both inner/outer BG coils are summarized in Table 2. Figure 1 shows a photograph of the solenoid test coils, including the GdBCO CC coils and BG coils used in this study.

A schematic drawing of the experimental setup can be seen in Fig. 2. The BG coils were connected with an AC power source to generate an external AC magnetic field. The tests for the elimination of SCIF were conducted in the following steps: 1) placing the INS or NI coil coaxially between the two BG coils connected in series while maintaining a gap of $5 \mathrm{~mm}, 2$ ) charging the INS or NI coil up to $50 \mathrm{~A}$ at a ramping rate of $2 \mathrm{~A} / \mathrm{s}$ in a bath of liquid nitrogen at $77 \mathrm{~K}, 3)$ applying various $\mathrm{AC}$ currents $\left(I_{\mathrm{AC}}\right)$ to the BG coils at a frequency of $100 \mathrm{~Hz}$ for $300 \mathrm{~s}$ to generate an external AC magnetic field, 4) discharging the INS or NI coil with subsequent warming to room temperature for complete elimination of the SCIF, and 5) repetition of steps 1-4 with increasing $I_{\mathrm{AC}}$. The magnetic field at the center of the coils $\left(B_{\mathrm{Z}}\right)$ was continuously monitored and recorded by a data acquisition (DAQ) system.

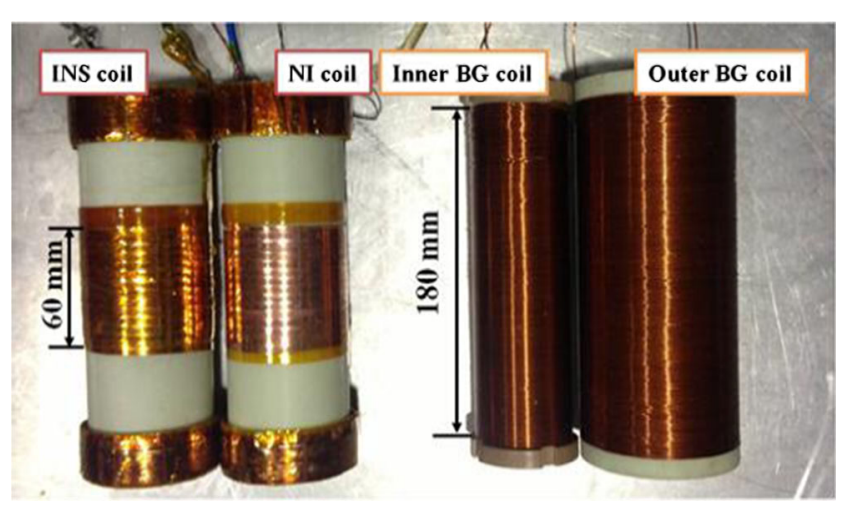

Fig. 1 Photograph of the test coils: GdBCO CC coils (left) and BG coils (right) 


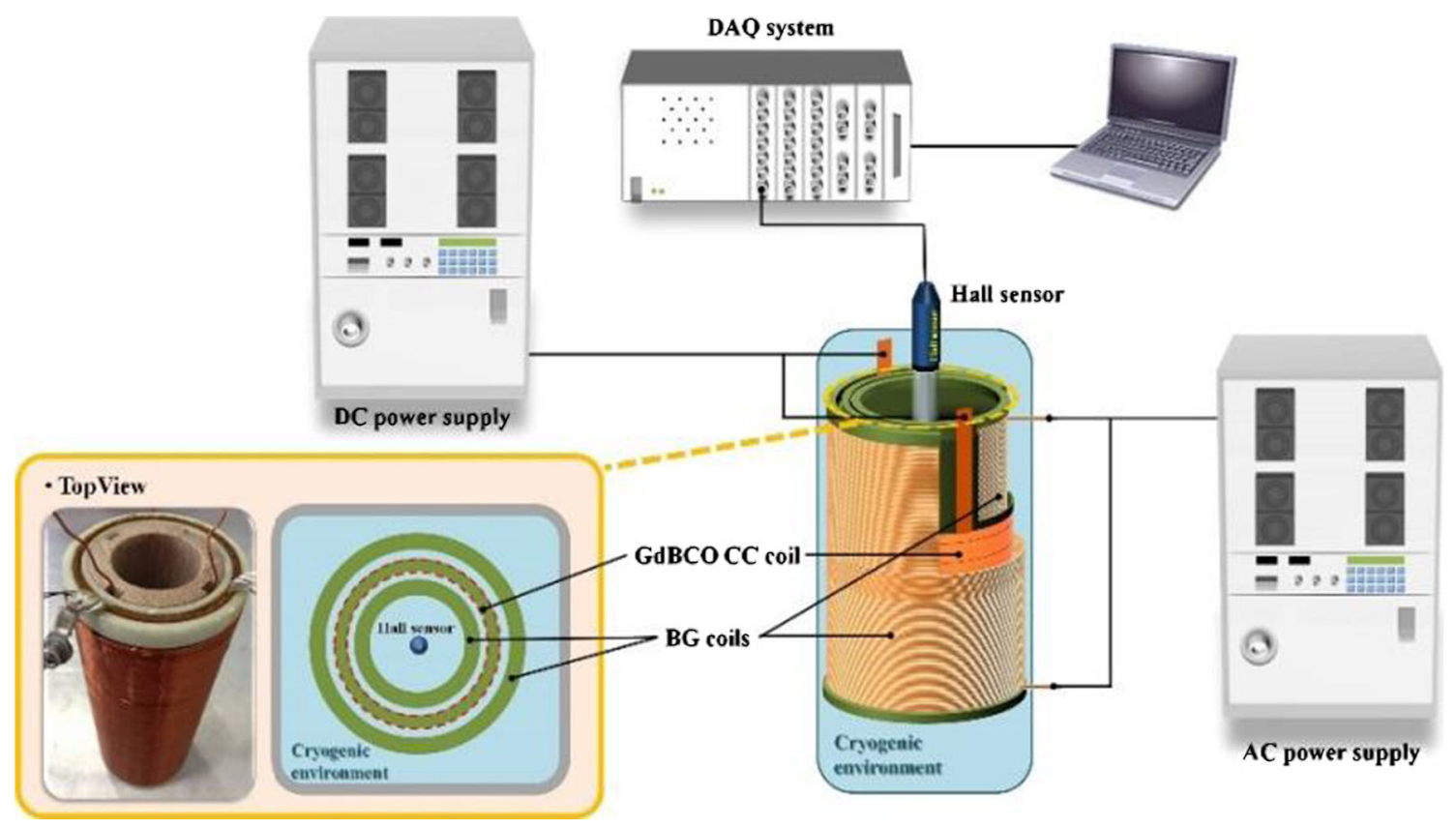

Fig. 2 Schematic drawing of the experimental setup

\section{Vortex Shaking Effect}

Figure 3a shows a schematic drawing of the screening current and magnetic field distribution within the GdBCO CC. When the GdBCO CC coil is charged by a direct transport current $\left(I_{\mathrm{DC}}\right)$, the radial DC field $\left(B_{\mathrm{DC}}\right)$ perpendicularly penetrating through the flat surface of the $\mathrm{CC}$ induces the screening current in such a direction as to oppose $B_{\mathrm{DC}}$ penetration. Consequently, the screening current which permanently flows in the superconductor, i.e., the supercurrent, leads to magnetization in the opposite direction of the $B_{\mathrm{DC}}$. As shown in Fig. 3b, when the alternating current $\left(I_{\mathrm{AC}}\right)$ is supplied to the $\mathrm{BG}$ coils, the axial $\mathrm{AC}$ magnetic field $\left(B_{\mathrm{AC}}\right)$ becomes dominant due to cancellation of the radial $B_{\mathrm{AC}}$ generated by reversely wound BG coils In the superconducting layer, circulation of the screening current is compelled to compensate for the axially oscillating $B_{\mathrm{AC}}$. Such a screening current causes the superconductor to become magnetized axially, leading to elimination of the SCIF generated by $B_{\mathrm{DC}}$ because the axial magnetization hardly affects the center field $[17,18]$. This change of magnetization induced by the $B_{\mathrm{DC}}$ reversing polarity into the axial direction perpendicular to the self-field of the coil is called the vortex shaking effect.

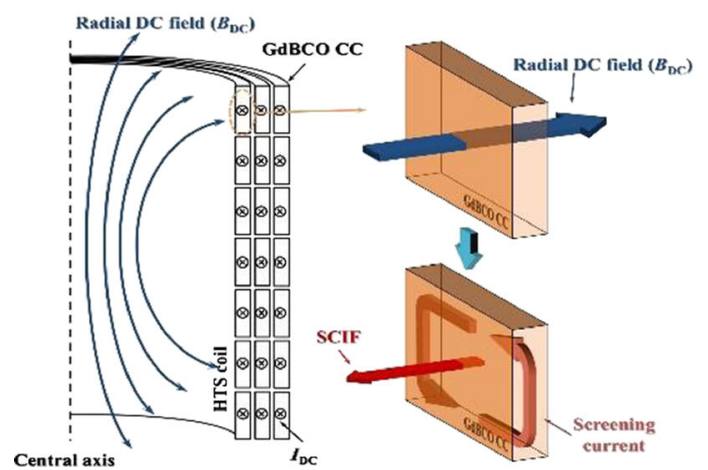

(a)

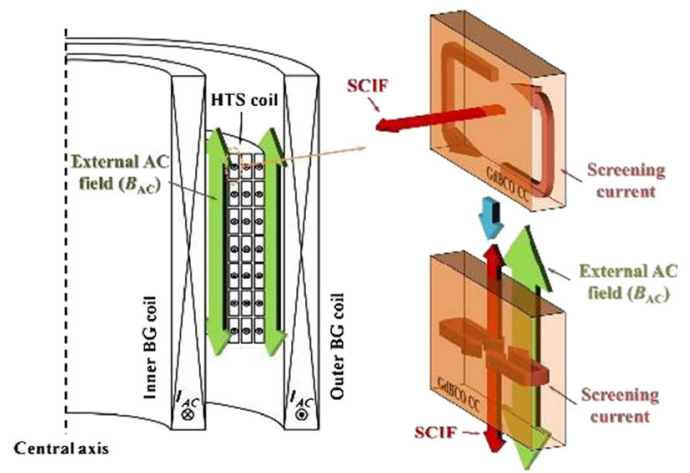

(b)

Fig. 3 Schematic drawing of the screening current and magnetic field distribution after applying the $B_{\mathrm{DC}}(\mathbf{a})$ and $B_{\mathrm{AC}}(\mathbf{b})$ 


\section{Results and Discussion}

\subsection{Determination of the SCIF in the NI Coil}

The SCIF in a conventionally insulated coil can be readily determined after the ramping current reaches the target current. However, in the case of NI coils, in which turn-to-turn insulation was completely eliminated, the resulting charging delays prove to be an obstacle to the clarification of SCIF magnitude. The delays observed in NI coils are caused by the radial current path through the non-insulated turnto-turn contacts. During charging of the supply current, the inductance voltage compels the supply current to become bifurcated into radial and spiral current flows. Thus, the spiral current lags behind the supply current, consequently leading to the charging delays typically observed in NI coils [9-16]. For measurement of the SCIF, even after coils come under steady-state operation, the field error caused by delays could be subsumed in the experimentally measured $B_{\mathrm{Z}}$, as well as the SCIF in the NI coils. Hence, to eliminate the field delay from the measured $B_{\mathrm{Z}}$ traces, the charging characteristics of NI coils should be calculated using the equivalent circuit model (see Fig. 4). By applying Kirchhoff's laws, the equivalent circuit model can be expressed by the following equation:

$L_{c o i l} \frac{d\left(I_{s c}\right)}{d t}+V_{c}\left(\frac{I_{s c}}{I_{c}}\right)^{n}=R_{c}\left(I_{o p}-I_{s c}\right)$.

In order to obtain the $R_{\mathrm{c}}$ of the NI coil for the calculation of $B_{\mathrm{Z}}(t)$, sudden discharge tests were carried out in a bath of

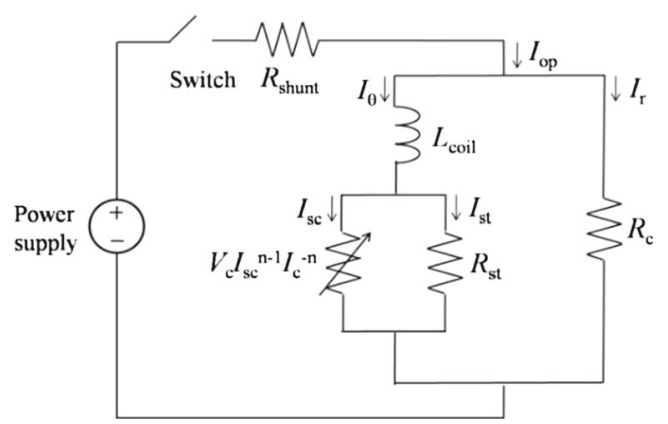

Fig. 4 Equivalent circuit model of the NI test coil connected with a DC power supply, a shunt resistor, and a switch [16]. $L_{\text {coil }}$ denotes the self-inductance of the coil, while $R_{s t}$ is the stabilizer resistance, $I_{c}$ is the coil's critical current, $V_{c}$ is the voltage criterion for $I_{c}, n$ is the $n$ value, and $I_{o p}$ is the current input from the power supply. $I_{\theta}$ and $\mathrm{I}_{\mathrm{r}}$ are the currents flowing through the spiral and radial directions of the coil, respectively. $I_{s c}$ and $I_{s t}$ are the currents flowing through the superconductor and stabilizer, respectively. $R_{c}$ is the characteristic resistance of the NI coil, representing the sum of the resistances in the radial direction liquid nitrogen at $77 \mathrm{~K}$. During the tests, $I_{\mathrm{op}}$ was kept constant so that the coil would be under steady-state operation before $I_{\mathrm{op}}$ was cut off.

Figure 5 shows the result of the sudden discharge test at $50 \mathrm{~A}$ for the NI coil. To facilitate comparison, each $B_{\mathrm{Z}}$ was normalized to its initial value. The decay time constants $(\tau)$ of the NI coil, defined as the time at the normalized $B_{\mathrm{Z}}$ of 0.37 , were obtained as 5.35 . The $R_{\mathrm{c}}$ of the NI coil was then calculated to be $17.68 \mu \Omega$, using the following equation:

$R_{\mathrm{c}}=\frac{L_{\text {coil }}}{\tau}$

The $B_{\mathrm{Z}}$ of the NI coil under the charging conditions could be simulated by applying the $R_{\mathrm{c}}$ calculated based on the equivalent circuit model.

The measured and simulated $B_{\mathrm{Z}}$ traces of the NI coil after the charging test at $50 \mathrm{~A}$, with a ramping rate of $2 \mathrm{~A} / \mathrm{s}$, are shown in Fig. 6. The simulated $B_{\mathrm{Z}}$ of the NI coil increased logarithmically to reach $30.84 \mathrm{mT}$ at $c a .63 \mathrm{~s}$, which was $38 \mathrm{~s}$ later than the time when the coil actually came under steady-state operation (25 s).

However, the measured $B_{\mathrm{Z}}$ still remained below 30.84 $\mathrm{mT}$ even at $80 \mathrm{~s}$, exhibiting a SCIF of $\mathrm{ca} .-0.84 \mathrm{mT}$. Note that the SCIF at the center of the energized coil had the opposite polarity to the magnetic field generated by the supply current [23]. The test results indicated that the SCIF in the NI coil could be defined at the time reference $38 \mathrm{~s}$ past the start of steady-state operation, as the charging delays were clearly diminished at that time.

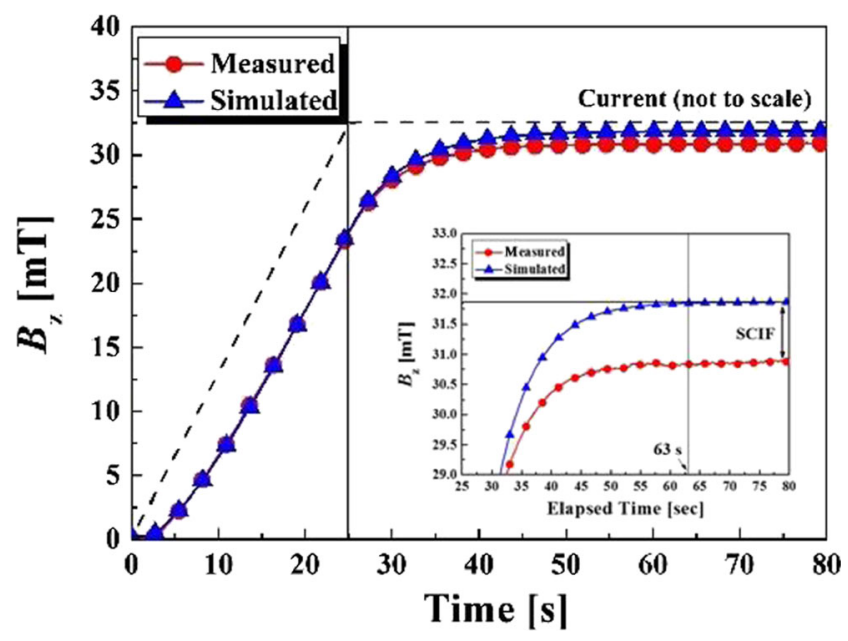

Fig. 5 Normalized $B_{\mathrm{Z}}$ traces of the NI coil, simulated and then measured by the sudden discharging test 


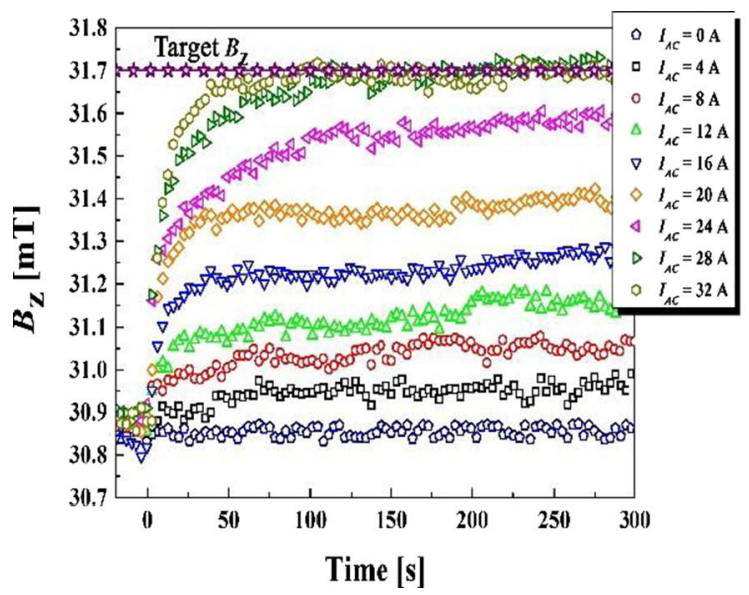

(a)

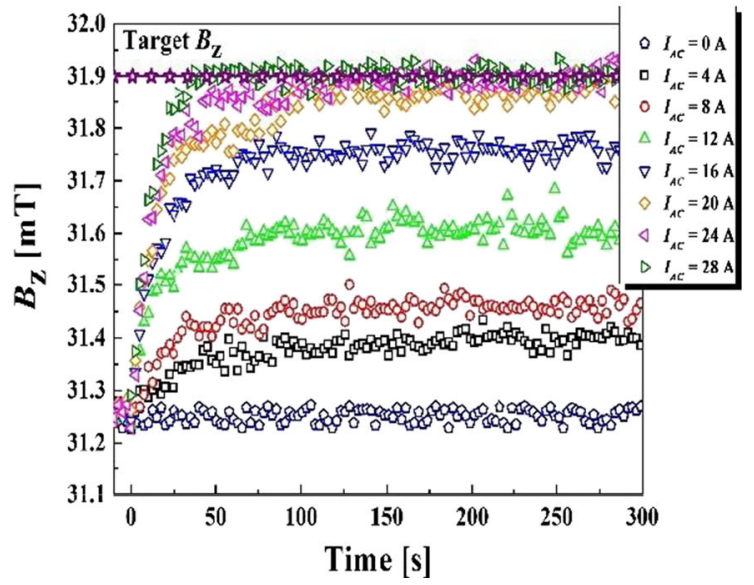

(b)

Fig. $6 B_{\mathrm{Z}}$ traces of the NI coil obtained from the charging test at $50 \mathrm{~A}$ with charging rates of $2 \mathrm{~A} / \mathrm{s}$. The inset graph provides an enlarged view for easy comparison

4.2 The Effects of the External AC Magnetic Field on SCIF in INS and NI Coils

Figure 7 shows the axial center field of the INS and NI coils with respect to the amplitude of the $I_{\mathrm{AC}}$. The target $B_{\mathrm{Z}}$ in the figures represent the $B_{\mathrm{Z}}$ values obtained numerically using the equivalent circuit model presented in Fig. 4, which are proportional to the magnet constant $\left(B_{Z} / \mathrm{A}\right)$. During charging of the INS coil at $50 \mathrm{~A}$, the $B_{\mathrm{Z}}$ without the application of an external AC field (i.e., $I_{\mathrm{AC}}=0 \mathrm{~A}$ ) was $30.84 \mathrm{mT}$, corresponding to the SCIF of ca. $0.84 \mathrm{mT}$. When the INS coil was exposed to an external AC field, the $B_{z}$ increased logarithmically, i.e., the SCIF decreased exponentially. The measured $B_{\mathrm{Z}}$ values of the INS coil at $I_{\mathrm{AC}}=4,8,12,16$, 20, 24, and 28 A were 30.95, 31.08, 31.15, 31.29, 31.41,

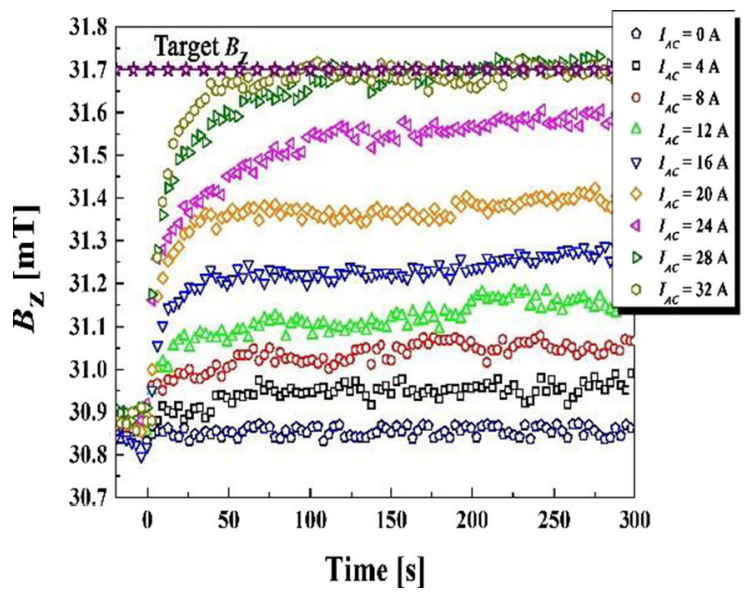

(a)
31.56, and $31.70 \mathrm{mT}$, respectively. As expected, the SCIF of the INS coil linearly decreased with increasing $I_{\mathrm{AC}}$. However, the $B_{\mathrm{Z}}$ trace at $I_{\mathrm{AC}}=32 \mathrm{~A}$ was almost identical to that at $I_{\mathrm{AC}}=28 \mathrm{~A}$, implying extinction of the SCIF. This means that the orientation of the GdBCO magnetization in the INS coil was completely changed into the axial direction.

As shown in Fig. 7b, the SCIF of the NI coil at $I_{\mathrm{AC}}=$ 0 A was $c a$. $-0.64 \mathrm{mT}$, which was $24 \%$ lower than that of the INS coil at $I_{\mathrm{AC}}=0 \mathrm{~A}$. This result implied that a screening current induced by the $B_{\mathrm{DC}}$ could flow not only along its original path in the winding direction, but also through the turn-to-turn and layer-to-layer contacts in the axial and radial directions. This suggested that the screening current may decay through contact resistance, resulting in a lower SCIF compared to the INS coil.

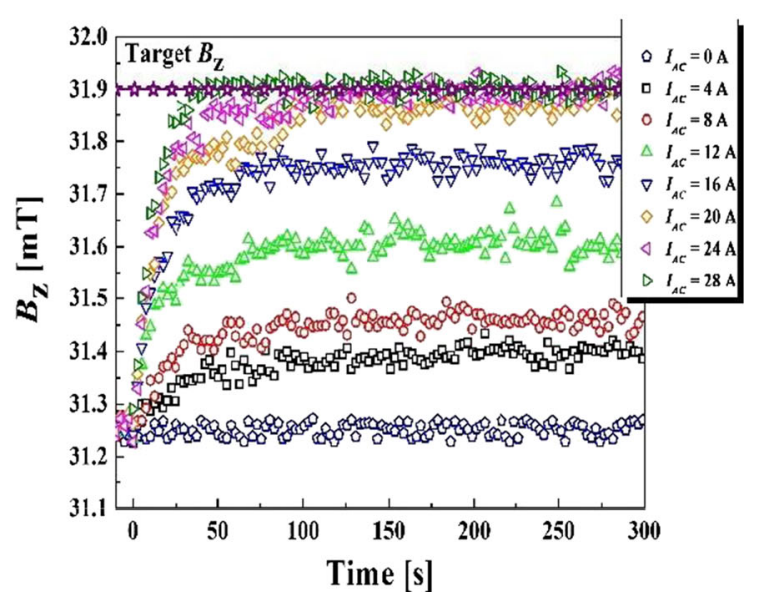

(b)

Fig. $7 \quad B_{\mathrm{Z}}$ traces of INS (a) and NI (b) coils, with respect to varying $I_{\mathrm{AC}}$ 
The SCIFs in the NI coil exhibited similar behavior to those in the INS coil under the external AC magnetic field. The $B_{z}$ values of the NI coil at $300 \mathrm{~s}$ for $I_{\mathrm{AC}}=4,8,12$, 16 , and 20 A were $31.39,31.47,31.61,31.78$, and 31.86 $\mathrm{mT}$, respectively. However, further increase of the $I_{\mathrm{AC}}$ led to saturated fields of $31.89 \mathrm{mT}$ at $24 \mathrm{~A}$ and $31.89 \mathrm{mT}$ at $28 \mathrm{~A}$. The field saturation of the NI coil observed at a lower external $\mathrm{AC}$ field resulted from the lower value of the SCIF compared to the INS coil. Consequently, the SCIFs in the NI coil could be completely eliminated, even at lower $I_{\mathrm{AC}}$ compared to the INS coil, enabling the practical utilization of NI magnets in high-resolution NMR spectrometers.

\section{Conclusion}

In order to eliminate the screening current-induced fields (SCIF) in GdBCO CC coils by exposing them to external AC magnetic fields, fully insulated (INS) and no-insulation (NI) GdBCO CC coils were fabricated for testing. Prior to investigating the effects of an external $\mathrm{AC}$ magnetic field on the SCIF, the magnetic flux density $\left(B_{\mathrm{Z}}\right)$ of the coil was calculated using the equivalent circuit model, and compared to the $B_{\mathrm{Z}}$ obtained empirically to quantify the SCIF in the INS and NI coils The value of the SCIF measured for the NI coil $(-0.64 \mathrm{mT})$ was $24 \%$ smaller than that in the INS coil $(-0.84 \mathrm{mT})$ due to the current paths originating from the turn-to-turn and layer-to-layer contacts, suggesting that the screening current dissipated through contact resistance during charging As both the INS and NI coils were exposed to an external $\mathrm{AC}$ magnetic field, the $B_{\mathrm{Z}}$ of the coils increased gradually to eventually saturate at the theoretical values of 31.7 and $31.9 \mathrm{mT}$, respectively. This indicated full elimination of the SCIF, because of a change in the orientation of GdBCO magnetization (vortex shaking effect). In addition, the SCIF in the NI coil could be removed completely under lower external AC magnetic fields compared to the INS coil, due to the lower SCIF in the NI coil after charging. The experimental results were also in good agreement with the simulations, demonstrating the validity of the equivalent circuit model. Overall, the SCIF in the NI coil could be readily removed during exposure of the $\mathrm{GdBCO} \mathrm{CC}$ coils to the external AC magnetic field, resulting in improvement of the temporal stability of the NI HTS coil, thus guaranteeing the applicability of NI coils for practical high-resolution NMR spectrometers.
Acknowledgments This work was supported by the KETEP grant from the International Collaborative R\&D Program, funded by the Korean government MKE (20118520020020). This work was supported by the Mid-Career Researcher Program through an NRF grant funded by the MEST (2012-046999).

\section{References}

1. Iwasa, Y.: Physica C 445-448, 1088-1094 (2006)

2. Kiyoshi, T., Choi, S., Matsumoto, S., Zaitsu, K., Hase, T., Miyazaki, T., Otsuka, A., Yoshikawa, M., Hamada, M., Hosono, M., Yanagisawa, Y., Nakagome, H., Takahashi, M., Yamazaki, T., Maeda, H.: IEEE Trans. Appl. Supercond. 20, 714-717 (2010)

3. Otsuka, A., Kiyoshi, T., Matsumoto, S., Kominato, K., Takeda, M.: IEEE Trans. Appl. Supercond. 18, 852-855 (2008)

4. Ahn, M.C., Yagai, T., Hahn, S.Y., Ando, R., Bascuñán, J., Iwasa, Y.: IEEE Trans. Appl. Supercond 19, 2269-2272 (2009)

5. Yang, D.G., Kim, K.L., Choi, Y.H., Kwon, O.J., Park, Y.J., Lee, H.G.: Supercond. Sci. Technol. 26, 105025 (2013)

6. Amemiya, N., Akachi, K.: Supercond. Sci. Technol. 21, 095001 (2008)

7. Gu, C., Qu, T., Han, Z.: IEEE Trans. Appl. Supercond. 17, 2394 2397 (2007)

8. Ahn, M.C., Jang, J., Lee, W.S., Hahn, S.Y., Lee, H.G.: IEEE Trans. Appl. Supercond. 24, 4301605 (2014)

9. Hahn, S.-Y., Park, D.K., Bascuñán, J., Iwasa, Y.: IEEE Trans. Appl. Supercond. 21, 15921595 (2011)

10. Kim, Y.G., Hahn, S.-Y., Kim, K.L., Kwon, O.J., Lee, H.G.: IEEE Trans. Appl. Supercond. 22, 5200604 (2012)

11. Kwon, O.J., Kang, D.H., Kang, J.S., Ko, T.K., Lee, H.G.: Supercond. Sci. Technol. 26, 085025 (2012)

12. Kim, K.L., Choi, Y.H., Yang, D.G., Song, J.B., Lee, H.G.: Supercond. Sci. Technol. 27, 015001 (2013)

13. Choi, S., Jo, H.C., Hwang, Y.J., Hahn, S., Ko, T.K.: IEEE Trans. Appl. Supercond. 22, 4904004 (2012)

14. Hahn, S.Y., Kim, Y., Ling, J., Voccio, J., Park, D.K., Bascuñán, J., Shin, H.J., Lee, H.G., Iwasa, Y.: IEEE Trans. Appl. Supercond. 23, 4601705 (2013)

15. Wang, X., Hahn, S.Y., Kim, Y., Bascuñán, J., Voccio, J., Lee, H.G., Iwasa, Y.: Supercond. Sci. Technol. 26, 035012 (2013)

16. Kim, Y.G., Yang, D.G., Lee, J.S., Kim, W.J., Kim, S.H., Lee, H.G.: IEEE Trans. Appl. Supercond. 24, 4900404 (2014)

17. Kajikawa, K., Funaki, K.: Supercond. Sci. Technol. 24, 125005 (2011)

18. Kajikawa, K., Funaki, K.: IEEE Trans. Appl. Supercond. 22, 4400404 (2012)

19. Mikitik, G.P., Brandt, E.H.: Phys. Rev. B 67, 104511 (2003)

20. Brandt, E.H., Mikitik, G.: Supercond. Sci. Technol. 17, S1-S5 (2004)

21. Brandt, E.H., Mikitik, G.P.: J. Low Temp. Phys. 139, 221-227 (2005)

22. Yanagisawa, Y., Kominato, Y., Nakagome, H., Hu, R., Takematsu, T., Takao, T., Uglietti, D., Kiyoshi, T., Takahashi, M., Maeda, H.: IEEE Trans. Appl. Supercond. 21, 1640-1643 (2011)

23. Yanagisawa, Y., Nakagome, H., Koyama, Y., Hu, R., Takao, T., Hamada, M., Kiyoshi, T., Takahashi, M., Maeda, H.: Physica C 469, 1996-1999 (2009) 